4 Heading RC. Proton pump inhibitor failure in gastro-oesophageal reflux disease: a perspective aided by the Gartner hype cycle. Clin Med 2017;17:132-6.

5 Black D. The new UK internal medicine curriculum. Clin Med 2017;17:103-4.

6 Halter M, Wheeler C, Drennan VM et al. Physician associates in England's hospitals: a survey of medical directors exploring current usage and factors affecting recruitment. Clin Med 2017;17:126-31.
7 Peters K, Smith J. The Francis Crick Institute. Clin Med 2017;17:105-7.

8 Maher D, Ford N, Gilmore I. Practical steps in promoting synergies between clinical medicine and public health. Clin Med 2017;17:100-2.

\title{
Practical steps in promoting synergies between clinical medicine and public health
}

\author{
Authors: Dermot Maher, ${ }^{A}$ Nathan Ford ${ }^{B}$ and Ian Gilmore ${ }^{C}$
}

\section{Introduction}

The dichotomy between clinical medicine and public health in the UK and elsewhere often starts at medical school and continues throughout postgraduate training. However, there is increasing recognition of the benefits of bridging this often deep divide, ${ }^{1}$ most importantly for people's health. Without effective efforts to address the 'upstream' determinants of health and to improve secondary prevention, physicians will face even greater challenges in managing ever-increasing service demands. ${ }^{2}$ Based on global health experience, areas where the practice of clinical medicine can benefit public health include disease control, advocacy and ethics, while areas where good public health practice benefits clinical medicine include routine use of data to improve care delivery and the establishment of simplified and standardised approaches in support of extending the benefits of available treatments as widely, equitably and efficiently as possible. ${ }^{1}$

Dr Tom Frieden, the director of the US Centers for Disease Control, has described the healthcare and public health fields as 'inevitably and increasingly interdependent'. ${ }^{3}$ In a rapidly changing healthcare landscape in many countries,

Authors: ${ }^{\text {A }}$ coordinator, Special Programme for Research and Training in Tropical Diseases, World Health Organization, Geneva, Switzerland; ${ }^{\text {B }}$ scientist, Department of HIV and Global Hepatitis Programme, World Health Organization, Geneva, Switzerland; ${ }^{C}$ consultant physician and gastroenterologist, Royal Liverpool University Hospitals and honorary professor, Department of Medicine, University of Liverpool, UK there is also an increasing impetus for clinical academic centres to collaborate more widely with the aim of moving beyond individual patient care towards population health improvement. ${ }^{4}$ There is scope for clinicians (including academic clinicians) and public health practitioners to work together in ensuring complementarity between the two health fields and maximising the health gains from working together. In seeking the synergies between individual patient care and population health measures, the clinician's perspective on individual patient wellbeing can serve as a counterbalance to the public health concern with populations and attendant risk of subsuming individual wellbeing in the interests of 'the common good'. Conversely, the public health practitioner's perspective on population wellbeing can help to ensure that the benefits of consistently good clinical care are made widely and equitably available.

In the UK healthcare system, primary care is largely delivered through the GP system while secondary and tertiary care is delivered through the hospital system. The synergies between clinical medicine and public health are well established in primary care but much less so in secondary and tertiary care. The GP system integrates clinical care with preventive medicine and public health in delivering health services:

1 primary clinical care (patient-initiated consultations)

2 preventive medicine (eg cervical cancer screening)

3 public health (eg childhood immunisations).

While the hospital system provides some examples of synergies between clinical medicine and public health, there is plenty of scope for developing these synergies further in the interests of improved health. Building on examples in hospital care, we propose some practical steps to overcome the divide 
in the practice of clinical medicine and public health and obtain the health benefits of a 'clinical public health medicine' perspective.

\section{Public health delivery from hospital settings}

While a traditional view may be that public health activities should be delivered from community settings, this does not take into consideration the role - both actual and potential played by hospitals in public health and the opportunities for public health delivery from hospital settings. This can be approached from both directions, with public health activities embraced by hospital departments and hospital departments hosting public health activities. For example, a large cardiac department in a tertiary hospital is concerned with providing high-quality clinical care to individual patients and with ensuring that, from the public health point of view, the cardiological needs of the population served by the hospital are met effectively, efficiently and equitably. It would follow that, in this example, a cardiology department should have at least one cardiologist with an interest in the health of the community providing input on prevention strategies and health policies. Such an approach has recently been advocated to address the unmet need of cardiovascular disease (CVD) through the promotion of community activities, such as mobile phone text messaging to improve medication adherence, non-physician health workers for the delivery of community CVD care in areas of need and low-cost single-pill combination CVD therapy. $^{5}$

Regarding the hosting of public health activities by hospital departments, an example of the growing role for public health in acute hospitals is provided by the activities of the public health department for Barts Health NHS Trust based at the Royal London Hospital. This public health department is collaborating with the clinical departments in developing care pathways, analysing inequalities in access, referrals and outcomes, advising on trust policies on inequalities, and managing operational public health services - such as smoking cessation and screening. ${ }^{6}$ Such close collaborative arrangements between public health and hospital departments would also enable public health physicians to undertake session clinical work and keep their finger on the pulse of clinical care delivery. Another example of clear opportunities is liver disease. The three main causes of liver damage in the UK - alcohol, obesity and viral hepatitis - are all potentially avoidable or can be ameliorated by public health measures; community health would be enhanced if clinicians delivering services understood the underlying causes and were powerful advocates for improvements.

\section{Research}

The potential of harnessing data from medical records for research has been widely recognised. As an initiative of NHS England, Care.data aimed to ensure that more joined-up data are made available to clinicians, commissioners, researchers, charities and patients to improve the quality, safety and effectiveness of care services. This initiative has been described as potentially 'the best clinical laboratory in the world and the benefit would be felt first in the UK. This could change the game in health research and healthcare. ${ }^{7}$ Turning this ambition into practice, however, has proved more difficult than anticipated. Despite earlier warning signs, ${ }^{8}$ efforts to set up the scheme have now floundered through inadequate mobilisation of support among doctors working in the NHS and failure to gain public support (mainly because of insufficient reassurance about confidentiality). ${ }^{9}$ One of the lessons learned is the need for the research community to engage more effectively with other groups, such as the public health community, with relevant expertise in reaching out to the population at large and putting a persuasive case to link individual and population benefits. Practical steps to ensure close working relationships between researchers, clinicians and public health practitioners will help to promote research based on patients' data for individual and community benefit.

\section{Training}

Developments in undergraduate and postgraduate medical education and training need to meet evolving healthcare needs. Two important trends in modern medicine may be seen to run in opposite directions: first, the move towards 'precision medicine' with the aim of harnessing the potential of developments in genomics to improve individual care; and secondly, an increasing recognition of the interdependence of individual and community health, which is of particular importance in an ageing society. While the trend towards 'precision medicine' has received attention in reviews of undergraduate and postgraduate medical education and training, ${ }^{10}$ the trend of individual and community health interdependence has remained largely overlooked. Flexibility in training is highlighted as one of five key themes in the 2013 independent review of training commissioned by the General Medical Council, ${ }^{11}$ yet in practice individuals get trapped in narrow, specialty, GP or public health training with no meaningful opportunities for 'laddering across' between them. Meaningful flexibility would provide the opportunity to ensure the right balance of emphasis on clinical medicine and public health in undergraduate medical training and to systematise cross-over training and accreditation, ie accredited public health training for postgraduate trainees in clinical specialties and accredited clinical training for postgraduate trainees in public health.

\section{Conclusions}

Making progress in identifying and promoting synergies between clinical medicine and public health depends on close consultation and collaboration between key professional bodies, such as the Royal College of Physicians (RCP) and the Faculty of Public Health (FPH), supported by inspiring role models for the power of epidemiology and public health in our medical student and postgraduate training. The RCP and the FPH are well placed to work together to build the evidence base on ways of promoting and obtaining synergies between clinical medicine and public health. This involves identifying the range of activities (such as the initiative at the Royal London Hospital) aimed at maximising the synergies between clinical medicine and public health and developing and maintaining a compendium of initiatives. Locally successful activities can be further developed and expanded on a wider scale, while the national bodies responsible for training ensure that hospital 
specialists and public health physicians-in-training have real opportunities to develop complementary skills.

\section{Conflicts of interest}

The authors have no conflicts of interest to declare.

\section{Author contributions}

$\mathrm{DM}, \mathrm{NF}$ and IG all contributed to the conception and writing of the paper.

\section{Acknowledgements}

The views expressed by DM and NF are those of the authors and may not necessarily represent the views of the World Health Organization.

\section{References}

1 Maher D, Ford N. Promoting synergies between clinical medicine and public health. Trop Med Int Health 2016;21:938-42.

2 Ashton J. The state of public health services in England - why hospital physicians should be worried. Clin Med 2016;16:309-10.

3 Frieden TR. Shattuck lecture: The future of public health. $N$ Engl J Med 2015;373:1748-54.

4 Washington AE, Coye MJ, Boulware LE. Academic health systems' third curve: population health improvement. JAMA 2016;315: $459-60$.
5 Nieuwlaat R, Schwalm JD, Khatib R, Yusuf S. Why are we failing to implement effective therapies in cardiovascular disease? Eur Heart $J$ 2013;34:1262-9.

6 Middleton J. From the president. FPH ebulletin. London: Faculty of Public Health, 2016. http://us12.campaign-archive1.com/?u=9e81 8256890 cafc10f8039758\&id $=14796 \mathrm{~cd} 87 \mathrm{e} \& \mathrm{e}=[\mathrm{UNIQID}]$ [Accessed 13 February 2017].

7 Smyth C. Sharing medical data would turn UK into 'best clinical laboratory in world'. London: The Times, 2014. http://www.thetimes. co.uk/tto/health/news/article4040095.ece [Accessed 25 January 2017].

8 Jones G. Open wide. BMJ 2014:348:g1670.

9 Godlee F. What can we salvage from Care.data? BMJ 2016; 354:i3907.

10 Tooke J. Postgraduate medical education and training in the UK. BMJ 2013;347:f7604.

11 Greenaway D. Securing the future of excellent patient care. Final report of the independent review led by Professor David Greenaway. London: Shape of Training, 2013.

Address for correspondence: Dr Dermot Maher, Special Programme for Research and Training in Tropical Diseases, World Health Organization, 20 Avenue Appia, 1211 Geneva, Switzerland.

Email:maherd@who.int 